\title{
elsevier_YEESA_5620
}

\section{Impact of UV-B on drought- or cadmium-induced changes in the fatty acid composition of membrane lipid fractions in wheat}

givennameOrsolya Kinga Gondorsurname

givennameGabriella Szalaisurname

givennameViktória Kovácssurname

givennameTibor Jandasurname

givennameMagda Pálsurname*

pal.magda@agrar.mta.hu

Agricultural Institute, Centre for Agricultural Research, Hungarian Academy of Seienees,Sciences, H-2462 Martonvásár, POB 19, Hungary

${ }^{*}$ Corresponding author. Fax: +36 22569576 .

\section{Abstract}

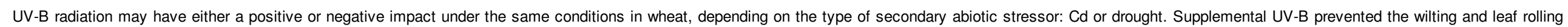

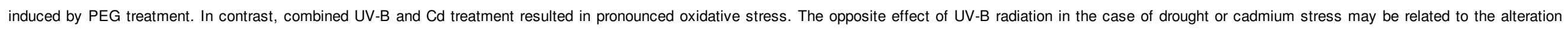

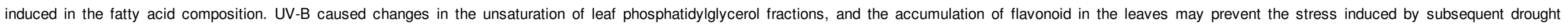

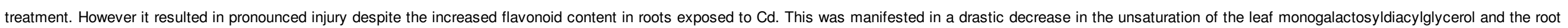

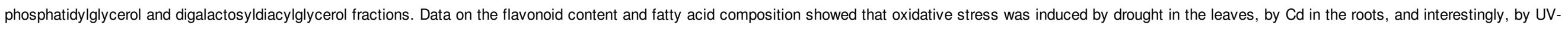

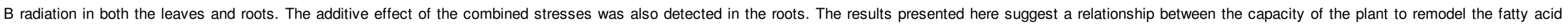
composition and its resistance to various stress factors.

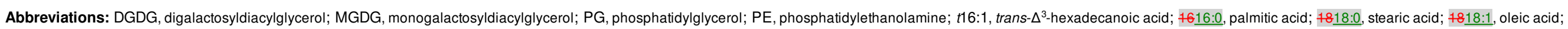
7818:2, linoleic acid; 7818:3, linolenic acid.acid

Keywords: Cadmium; Drought; Fatty acid composition; Flavonoids; UV-B radiation; Wheat

\section{Introduction}

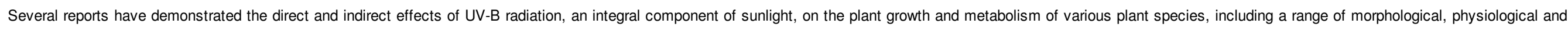

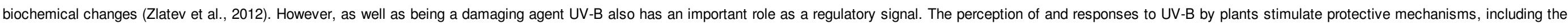

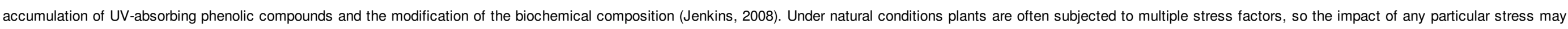

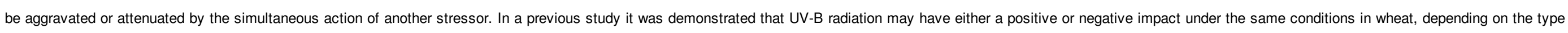

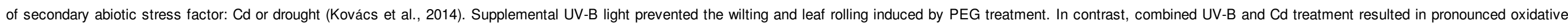

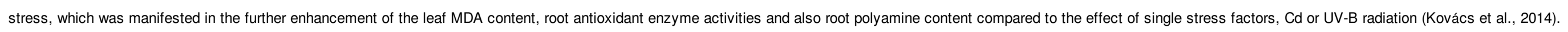

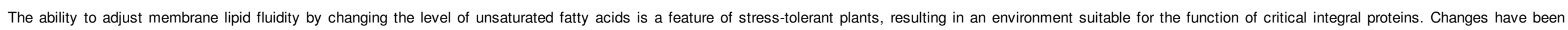

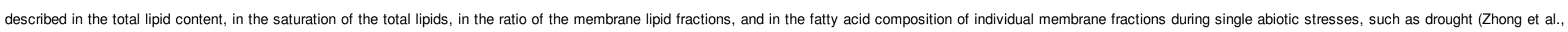

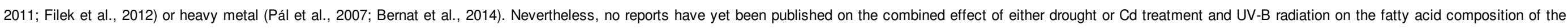
individual membrane fractions in wheat plants. 


\section{elsevier_YEESA_5620}

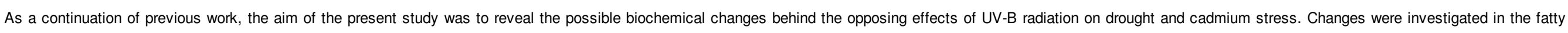

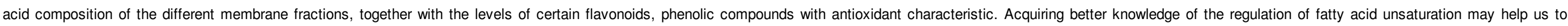
understand plant adaptation to changing environmental conditions.

\section{Materials and methods}

\subsection{Plant material and growth conditions}

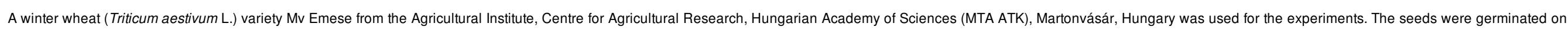

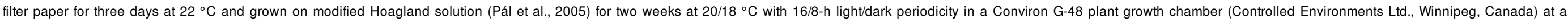

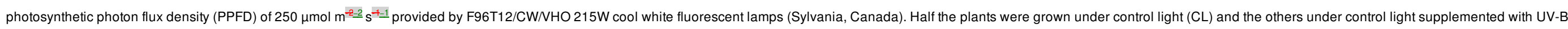

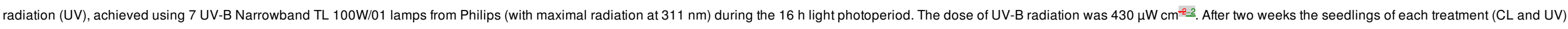

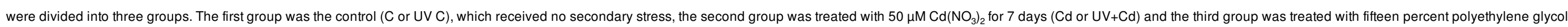

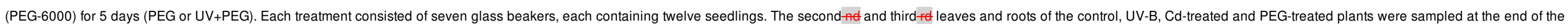
experiment.

\subsection{Lipid extraction and fatty acid analysis}

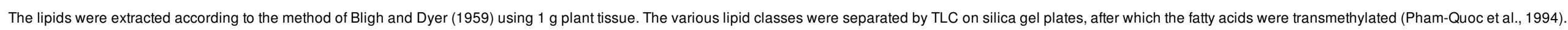

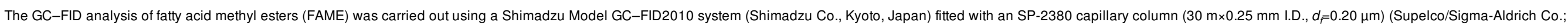

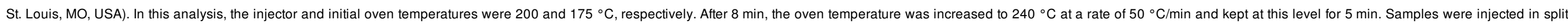
mode with a $1 / 5$ split ratio. The He carrier flow-rate was $37.8 \mathrm{~cm} / \mathrm{s}$. Heptadecanoic acid was used as internal standard for the quantification. Integration was performed using GC solution software 2.10 (Shimadzu Co., Kyoto, Japan).

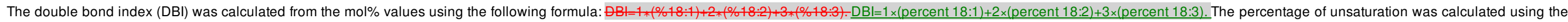

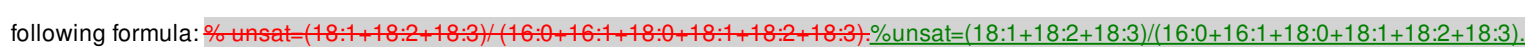

\subsection{Flavonoid extraction and analytical procedure}

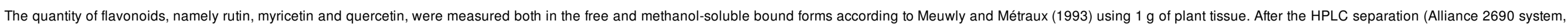

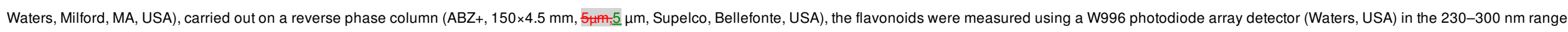

\subsection{Statistical analysis}

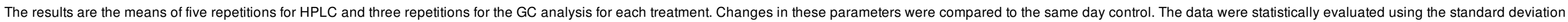
and $t$-test methods.

\section{Results}

\subsection{Fatty acid composition in different membrane fractions}

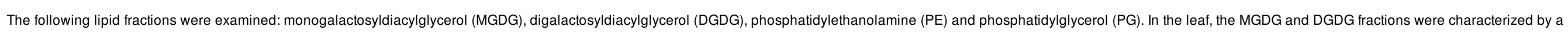

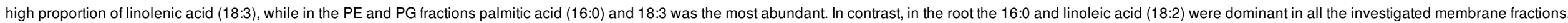

\subsubsection{Changes in leaf membrane fractions}

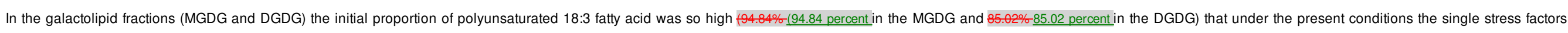




\section{elsevier_YEESA_5620}

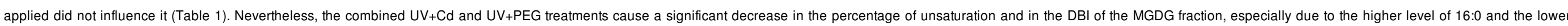
proportion of 18:3 (Table 1).

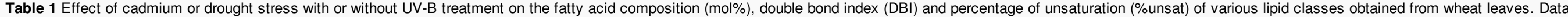
represent mean values $\pm \mathrm{SD}(n=3)$; different letters denote significant differences from control samples taken on the same day at $p<0.05$.

\begin{tabular}{|c|c|c|c|c|c|c|c|c|c|}
\hline Fraction & Treatment & C16:0 & $\mathrm{C} 16: 1$ & C18:0 & C18:1 & C18:2 & C18:3 & \%Unsat & $\mathrm{DBI}$ \\
\hline \multirow[t]{6}{*}{ MGDG } & CL C & $1.48 \pm 0.42 a$ & nd & $0.30 \pm 0.21 a$ & $0.29 \pm 0.11 a$ & $2.92 \pm 0.72 \mathrm{ab}$ & $94.84 \pm 1.50 \mathrm{~b}$ & $98.06 \pm 0.90 \mathrm{~b}$ & $290.67 \pm 8.90 \mathrm{~b}$ \\
\hline & CL Cd & $2.27 \pm 0.60 \mathrm{a}$ & nd & $0.55 \pm 0.22 a$ & $0.53 \pm 0.27 a$ & $3.55 \pm 0.53 \mathrm{~b}$ & $92.87 \pm 2.40 \mathrm{~b}$ & $96.96 \pm 1.16 \mathrm{~b}$ & $286.24 \pm 2.74 b$ \\
\hline & CL PEG & $2.40 \pm 0.75 a$ & nd & $0.81 \pm 0.51 \mathrm{ab}$ & $0.32 \pm 0.18 \mathrm{a}$ & $2.88 \pm 0.50 \mathrm{ab}$ & $93.59 \pm 1.81 \mathrm{~b}$ & $96.79 \pm 1.3 b$ & $286.84 \pm 5.62 b$ \\
\hline & UV C & $1.66 \pm 0.43 a$ & nd & $0.38 \pm 0.10 \mathrm{a}$ & $0.16 \pm 0.09 a$ & $2.41 \pm 0.22 \mathrm{a}$ & $95.39 \pm 3.70 \mathrm{~b}$ & $97.961 \pm 1.99 b$ & $291.15 \pm 2.41 b$ \\
\hline & $\mathrm{UV}+\mathrm{Cd}$ & $5.40 \pm 1.12 b$ & nd & $1.25 \pm 0.40 \mathrm{~b}$ & $0.56 \pm 0.23 a$ & $3.74 \pm 0.46 \mathrm{~b}$ & $87.25 \pm 2.12 a$ & $91.54 \pm 0.89 a$ & $269.78 \pm 5.75 a$ \\
\hline & $U V+P E G$ & $4.77 \pm 0.52 b$ & nd & $2.05 \pm 0.59 b$ & $0.64 \pm 0.4 a$ & $4.18 \pm 0.63 b$ & $88.29 \pm 4.20 a b$ & $93.10 \pm 2.05 a$ & $273.86 \pm 4.55 a$ \\
\hline \multirow[t]{6}{*}{ DGDG } & CL C & $9.69 \pm 0.98 \mathrm{ab}$ & nd & $1.51 \pm 0.41 \mathrm{ab}$ & $1.24 \pm 0.11 \mathrm{c}$ & $2.43 \pm 0.48 a$ & $85.02 \pm 3.52 \mathrm{ab}$ & $88.69 \pm 2.09 a$ & $261.15 \pm 5.63 a b$ \\
\hline & CL Cd & $8.26 \pm 0.75 a$ & nd & $1.15 \pm 0.32 a$ & $0.501 \pm 0.06 \mathrm{~b}$ & $2.76 \pm 0.46 a$ & $86.43 \pm 1.38 b$ & $89.69 \pm 1.98 a$ & $265.30 \pm 6.05 b$ \\
\hline & CL PEG & $10.48 \pm 1.74 a b$ & nd & $2.78 \pm 0.12 \mathrm{c}$ & $1.07 \pm 0.20 \mathrm{c}$ & $2.71 \pm 0.21 a$ & $82.43 \pm 3.55 a b$ & $86.38 \pm 1.98 a$ & $253.76 \pm 4.47 a$ \\
\hline & UV C & $10.51 \pm 0.81 \mathrm{~b}$ & nd & $2.18 \pm 0.48 \mathrm{bc}$ & $0.20 \pm 0.04 a$ & $2.31 \pm 1.14 a$ & $83.79 \pm 1.94 \mathrm{ab}$ & $86.29 \pm 1.32 a$ & $256.17 \pm 5.88 \mathrm{ab}$ \\
\hline & $\mathrm{UV}+\mathrm{Cd}$ & $11.38 \pm 1.34 b$ & nd & $1.85 \pm 0.55 \mathrm{ab}$ & $0.52 \pm 0.08 \mathrm{~b}$ & $2.72 \pm 0.97 a$ & $82.83 \pm 1.89 a$ & $86.07 \pm 3.69 a$ & $254.45 \pm 3.56 a$ \\
\hline & $U V+P E G$ & $11.50 \pm 1.90 \mathrm{~b}$ & nd & $1.98 \pm 0.56 \mathrm{abc}$ & $1.17 \pm 0.57 \mathrm{bc}$ & $3.16 \pm 1.01 \mathrm{a}$ & $81.74 \pm 1.26 \mathrm{a}$ & $86.07 \pm 2.94 a$ & $252.72 \pm 5.63 a$ \\
\hline \multirow[t]{6}{*}{ PE } & CL C & $33.48 \pm 3.61 \mathrm{~b}$ & nd & $8.56 \pm 2.02 \mathrm{c}$ & $4.07 \pm 1.64 \mathrm{c}$ & $21.66 \pm 1.52 a$ & $32.23 \pm 2.50 \mathrm{a}$ & $57.96 \pm 4.11 a$ & $144.08 \pm 9.06 a$ \\
\hline & CL Cd & $34.70 \pm 2.23 b$ & nd & $7.55 \pm 1.90 \mathrm{c}$ & $3.34 \pm 0.65 b c$ & $22.39 \pm 1.72 a b$ & $32.03 \pm 2.74 a$ & $57.75 \pm 5.66 \mathrm{a}$ & $144.21 \pm 6.26 \mathrm{a}$ \\
\hline & CL PEG & $30.63 \pm 3.65 \mathrm{ab}$ & nd & $3.42 \pm 1.60 \mathrm{ab}$ & $2.84 \pm 0.46 \mathrm{~b}$ & $20.65 \pm 2.80 a$ & $42.47 \pm 3.51 \mathrm{~b}$ & $65.95 \pm 4.27 a$ & $171.54 \pm 5.77 \mathrm{bc}$ \\
\hline & UV C & $25.90 \pm 2.30 \mathrm{a}$ & nd & $2.54 \pm 0.99 \mathrm{ab}$ & $2.92 \pm 0.55 b$ & $30.21 \pm 2.36 \mathrm{c}$ & $38.47 \pm 2.86 \mathrm{ab}$ & $71.57 \pm 3.33 b$ & $178.73 \pm 7.85 \mathrm{c}$ \\
\hline & $\mathrm{UV}+\mathrm{Cd}$ & $28.39 \pm 3.02 \mathrm{ab}$ & nd & $1.45 \pm 0.87 a$ & $1.79 \pm 0.32 a$ & $26.21 \pm 1.99 \mathrm{bc}$ & $42.16 \pm 3.00 \mathrm{~b}$ & $70.16 \pm 5.23 b$ & $180.68 \pm 9.01 c$ \\
\hline & $U V+P E G$ & $31.24 \pm 1.76 \mathrm{ab}$ & nd & $4.30 \pm 1.22 \mathrm{bc}$ & $2.0 \pm 0.56 \mathrm{ab}$ & $25.43 \pm 2.01 \mathrm{abc}$ & $37.03 \pm 2.69 \mathrm{ab}$ & $64.47 \pm 4.13 \mathrm{ab}$ & $163.97 \pm 2.25 b$ \\
\hline \multirow[t]{6}{*}{$P G$} & CL C & $24.24 \pm 1.33 \mathrm{~cd}$ & $13.97 \pm 1.31 a$ & $12.02 \pm 1.15 \mathrm{~d}$ & $2.14 \pm 0.26 \mathrm{c}$ & $2.81 \pm 0.16 a$ & $44.83 \pm 2.58 \mathrm{bc}$ & $49.77 \pm 1.75 b$ & $142.22 \pm 2.63 b$ \\
\hline & CL Cd & $17.25 \pm 1.65 a b$ & $24.13 \pm 2.01 b$ & $5.33 \pm 0.026 \mathrm{~b}$ & $3.06 \pm 0.96 \mathrm{~cd}$ & $7.08 \pm 0.70 \mathrm{~d}$ & $43.16 \pm 2.33 b$ & $53.29 \pm 1.66 \mathrm{bc}$ & $146.68 \pm 5.21 b c$ \\
\hline & CL PEG & $22.44 \pm 0.98 \mathrm{c}$ & $13.59 \pm 1.20 \mathrm{a}$ & $8.74 \pm 0.33 c$ & $3.50 \pm 0.69 d$ & $4.74 \pm 0.37 c$ & $46.99 \pm 2.10 \mathrm{bc}$ & $55.23 \pm 1.71 \mathrm{c}$ & $153.94 \pm 5.70 \mathrm{c}$ \\
\hline & UV C & $21.07 \pm 2.33 b c$ & $24.40 \pm 2.49 b$ & $8.01 \pm 0.46 \mathrm{c}$ & $1.37 \pm 0.32 b$ & $4.15 \pm 0.59 b$ & $38.92 \pm 0.80 a$ & $44.44 \pm 0.74 a$ & $126.43 \pm 7.20 \mathrm{a}$ \\
\hline & $U V+C d$ & $17.06 \pm 0.87 a$ & $25.66 \pm 2.11 \mathrm{~b}$ & $1.91 \pm 0.60 \mathrm{a}$ & $0.48 \pm 0.32 a$ & $6.12 \pm 0.15 d$ & $48.78 \pm 1.52 \mathrm{c}$ & $55.37 \pm 2.59 c$ & $159.04 \pm 4.71 \mathrm{c}$ \\
\hline & $U V+P E G$ & $25.97 \pm 1.26 \mathrm{~d}$ & $21.62 \pm 1.82 \mathrm{~b}$ & $5.84 \pm 0.75 b$ & $3.44 \pm 1.20 \mathrm{~cd}$ & $6.27 \pm 0.55 d$ & $36.86 \pm 2.62 a$ & $46.57 \pm 1.95 a$ & $126.56 \pm 3.99 a$ \\
\hline
\end{tabular}

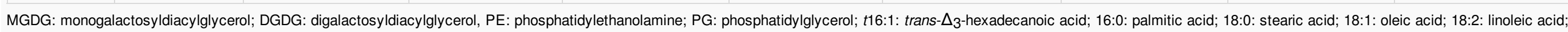
18:3: linolenic acid, nd: not detected. 


\section{elsevier_YEESA_5620}

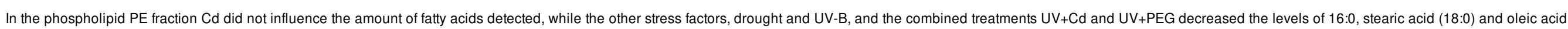
(18:1), but increased the proportion of 18:3 leading to an increase in DBI (Table 1).

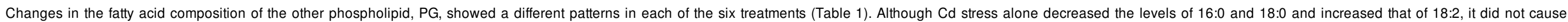

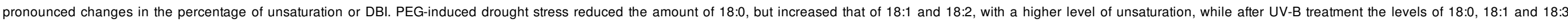

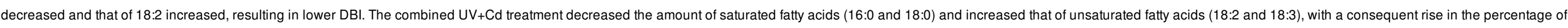

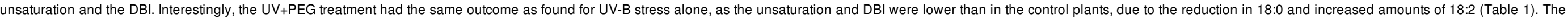
amount of $\Delta$ trans $-\Delta^{3}$-hexadecanoic acid (t16:1), which was only detectable in the leaf PG, increased significantly in all the treatments except for drought stress alone (Table 1).

\subsubsection{Changes in root membrane fractions}

In contrast to the leaves, significant changes were observed for all the lipid classes examined (Table 2).

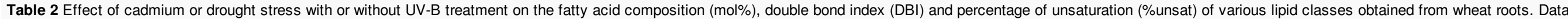
represent mean values $\pm \mathrm{SD}(n=3)$; different letters denote significant differences from control samples taken on the same day at $p<0.05$.

\begin{tabular}{|c|c|c|c|c|c|c|c|c|}
\hline Fraction & Treatment & $\mathrm{C} 16: 0$ & C18:0 & C18:1 & C18:2 & C18:3 & \%Unsat & $\mathrm{DBI}$ \\
\hline \multirow[t]{6}{*}{ MGDG } & CL C & $52.18 \pm 3.13 b$ & $15.22 \pm 1.46 a$ & $9.26 \pm 3.01 \mathrm{a}$ & $16.24 \pm 4.20 \mathrm{~b}$ & $7.10 \pm 1.37 a$ & $32.60 \pm 1.99 b$ & $63.04 \pm 4.63 b$ \\
\hline & $\mathrm{CL} \mathrm{Cd}$ & $43.38 \pm 1.66 a$ & $17.82 \pm 0.98 a$ & $16.35 \pm 1.88 \mathrm{~b}$ & $12.96 \pm 3.23 a b$ & $9.49 \pm 0.78 b$ & $38.80 \pm 1.75 \mathrm{c}$ & $70.73 \pm 2.39 \mathrm{bc}$ \\
\hline & CL PEG & $45.36 \pm 2.31 a$ & $17.83 \pm 0.66 \mathrm{a}$ & $7.61 \pm 0.39 a$ & $16.51 \pm 1.55 \mathrm{~b}$ & $12.69 \pm 2.72 b$ & $36.81 \pm 4.55 \mathrm{bc}$ & $78.69 \pm 6.82 \mathrm{c}$ \\
\hline & UV C & $52.42 \pm 2.10 \mathrm{~b}$ & $26.43 \pm 2.34 b$ & $6.26 \pm 1.11 a$ & $8.65 \pm 2.25 \mathrm{ab}$ & $6.25 \pm 1.13 a$ & $21.15 \pm 1.64 a$ & $42.29 \pm 3.21 a$ \\
\hline & $\mathrm{UV}+\mathrm{Cd}$ & $50.03 \pm 1.94 b$ & $17.20 \pm 2.64 a$ & $6.46 \pm 2.19 a$ & $15.58 \pm 3.69 a b$ & $10.74 \pm 0.99 b$ & $32.78 \pm 2.67 \mathrm{~b}$ & $69.84 \pm 2.37 \mathrm{bc}$ \\
\hline & $U V+P E G$ & $47.88 \pm 2.73 a b$ & $18.90 \pm 1.85 a$ & $6.24 \pm 1.37 a$ & $17.61 \pm 2.09 b$ & $9.38 \pm 0.87 \mathrm{~b}$ & $33.22 \pm 3.64 \mathrm{bc}$ & $69.58 \pm 5.67 \mathrm{bc}$ \\
\hline \multirow[t]{6}{*}{ DGDG } & CL C & $39.66 \pm 3.45 \mathrm{~cd}$ & $13.47 \pm 0.52 b$ & $8.48 \pm 0.33 b c$ & $28.31 \pm 1.89 b$ & $10.08 \pm 0.41 a$ & $46.87 \pm 3.46 \mathrm{~b}$ & $95.34 \pm 5.41 \mathrm{~b}$ \\
\hline & $\mathrm{CL} \mathrm{Cd}$ & $40.53 \pm 2.82 d$ & $12.48 \pm 0.12 a$ & $7.51 \pm 0.68 \mathrm{ab}$ & $25.49 \pm 3.56 \mathrm{~b}$ & $13.99 \pm 0.89 b$ & $46.99 \pm 0.92 b$ & $100.47 \pm 2.85 b$ \\
\hline & CL PEG & $27.27 \pm 0.0 \mathrm{~b}$ & $14.63 \pm 0.87 \mathrm{~b}$ & $10.21 \pm 1.25 \mathrm{c}$ & $31.73 \pm 2.19 \mathrm{~b}$ & $16.16 \pm 0.31 \mathrm{c}$ & $58.10 \pm 0.88 d$ & $122.14 \pm 7.42 \mathrm{c}$ \\
\hline & UV C & $34.760 \pm 0.68 \mathrm{~cd}$ & $12.82 \pm 0.70 \mathrm{ab}$ & $7.19 \pm 0.55 \mathrm{ab}$ & $13.50 \pm 0.96 a$ & $31.72 \pm 1.46 \mathrm{e}$ & $52.42 \pm 0.59 c$ & $129.36 \pm 6.34 \mathrm{c}$ \\
\hline & $\mathrm{UV}+\mathrm{Cd}$ & $44.88 \pm 2.72 d$ & $22.39 \pm 2.11 \mathrm{c}$ & $9.67 \pm 0.92 c$ & $14.32 \pm 1.28 a$ & $8.74 \pm 0.94 a$ & $32.73 \pm 0.64 a$ & $64.54 \pm 1.62 a$ \\
\hline & $U V+P E G$ & $20.70 \pm 1.40 \mathrm{a}$ & $12.46 \pm 0.82 a$ & $8.01 \pm 0.32 \mathrm{ab}$ & $39.07 \pm 3.08 \mathrm{c}$ & $19.76 \pm 1.54 d$ & $66.84 \pm 1.55 \mathrm{e}$ & $145.42 \pm 2.61 \mathrm{~d}$ \\
\hline \multirow[t]{6}{*}{$\mathrm{PE}$} & $\mathrm{CLC}$ & $31.87 \pm 1.52 b$ & $6.12 \pm 0.95 \mathrm{a}$ & $3.23 \pm 0.42 \mathrm{a}$ & $35.86 \pm 3.89 b$ & $22.91 \pm 1.26 \mathrm{ab}$ & $62.01 \pm 0.98 \mathrm{~b}$ & $143.70 \pm 1.26 \mathrm{~b}$ \\
\hline & $\mathrm{CL} \mathrm{Cd}$ & $30.17 \pm 1.37 \mathrm{~b}$ & $5.02 \pm 0.64 a$ & $5.24 \pm 0.15 b$ & $34.62 \pm 4.02 b$ & $24.94 \pm 1.96 \mathrm{bc}$ & $64.81 \pm 2.10 \mathrm{bc}$ & $149.31 \pm 6.91 b c$ \\
\hline & CL PEG & $23.02 \pm 0.32 a$ & $5.58 \pm 0.57 a$ & $5.33 \pm 0.82 \mathrm{bc}$ & $40.55 \pm 4.80 \mathrm{~b}$ & $25.52 \pm 1.55 \mathrm{bc}$ & $71.40 \pm 0.80 \mathrm{~d}$ & $162.99 \pm 14.52 \mathrm{~d}$ \\
\hline & UV C & $34.75 \pm 0.98 \mathrm{c}$ & $13.29 \pm 1.01 \mathrm{c}$ & $2.60 \pm 0.33 a$ & $27.51 \pm 1.96 a$ & $21.85 \pm 0.48 a$ & $51.96 \pm 2.14 a$ & $123.18 \pm 0.97 a$ \\
\hline & $\mathrm{UV}+\mathrm{Cd}$ & $30.98 \pm 1.26 \mathrm{~b}$ & $4.75 \pm 0.78 \mathrm{a}$ & $3.02 \pm 0.66 \mathrm{a}$ & $34.92 \pm 2.34 b$ & $26.33 \pm 1.16 \mathrm{c}$ & $64.27 \pm 2.40 \mathrm{bc}$ & $151.86 \pm 3.85 \mathrm{c}$ \\
\hline & $U V+P E G$ & $26.58 \pm 2.25 a$ & $8.27 \pm 0.61 b$ & $6.13 \pm 0.51 \mathrm{c}$ & $35.32 \pm 1.36 \mathrm{~b}$ & $23.69 \pm 0.96 \mathrm{~b}$ & $65.15 \pm 1.67 \mathrm{c}$ & $147.85 \pm 2.54 b c$ \\
\hline$P G$ & CL C & $42.00 \pm 2.23 a$ & $9.22 \pm 0.95 b$ & $9.83 \pm 0.95 \mathrm{c}$ & $19.67 \pm 3.81 \mathrm{~b}$ & $19.28 \pm 0.69 b$ & $48.79 \pm 1.38 b$ & $107.02 \pm 7.13 c$ \\
\hline
\end{tabular}




\section{elsevier_YEESA_5620}

\begin{tabular}{|c|c|c|c|c|c|c|c|}
\hline $\mathrm{CLCd}$ & $43.32 \pm 6.55 \mathrm{ab}$ & $24.39 \pm 1.78 \mathrm{c}$ & $8.74 \pm 0.56 \mathrm{c}$ & $9.26 \pm 2.59 a$ & $14.29 \pm 0.43 a$ & $32.29 \pm 4.18 \mathrm{a}$ & $70.13 \pm 10.84 a$ \\
\hline CL PEG & $41.25 \pm 7.51 \mathrm{ab}$ & $8.24 \pm 0.26 \mathrm{~b}$ & $5.67 \pm 0.53 b$ & $19.29 \pm 0.42 b$ & $25.56 \pm 0.47 d$ & $50.51 \pm 3.60 \mathrm{~b}$ & $120.92 \pm 5.96 \mathrm{~d}$ \\
\hline UV C & $46.81 \pm 0.81 \mathrm{~b}$ & $6.29 \pm 0.20 \mathrm{a}$ & $4.91 \pm 0.45 b$ & $19.83 \pm 0.02 b$ & $20.83 \pm 0.70 \mathrm{~b}$ & $46.19 \pm 0.85 b$ & $107.05 \pm 0.87 \mathrm{c}$ \\
\hline$U V+C d$ & $40.84 \pm 3.60 \mathrm{a}$ & $21.55 \pm 1.10 \mathrm{c}$ & $5.68 \pm 0.97 b$ & $10.86 \pm 1.84 a$ & $21.07 \pm 0.56 \mathrm{c}$ & $37.61 \pm 2.02 a$ & $90.62 \pm 1.98 \mathrm{~b}$ \\
\hline$U V+P E G$ & $41.34 \pm 2.39 a$ & $8.49 \pm 0.94 b$ & $4.02 \pm 0.27 a$ & $19.55 \pm 0.54 b$ & $26.59 \pm 0.47 d$ & $50.16 \pm 2.82 b$ & $122.89 \pm 2.67 d$ \\
\hline
\end{tabular}

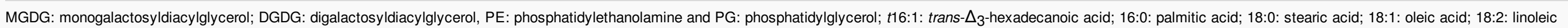
acid; 18:3: linolenic acid.

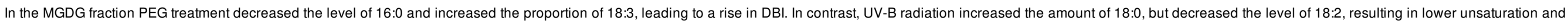
DBI.

Cd, alone or in combination with UV-B did not markedly influence the fatty acid pattern (Table 2).

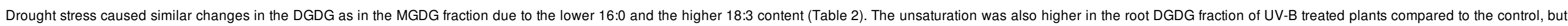

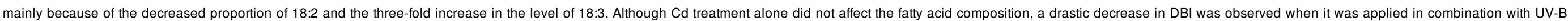
radiation due to an increase in 18:0, together with a decrease in 18:2 and 18:3. PEG and UV-B treatment were found to have an additive effect, leading to a high level of unsaturation (Table 2).

In the PE fraction, similarlysimilar to MGDG, the percentage of unsaturation and the DBI were increased by PEG, decreased by UV-B, while the combined UV+Cd treatment also caused slight increase (Table 2).

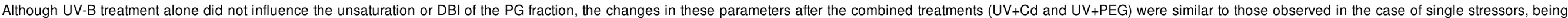

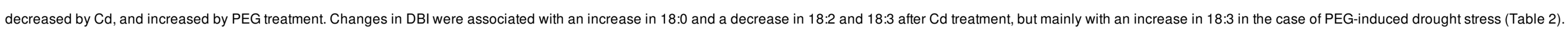

\subsection{Changes in flavonoid contents}

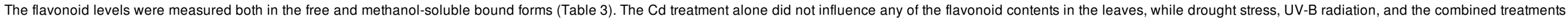
$(U V+C d$ and $U V+P E G)$ increased the total rutin, myricetin, and quercetin.

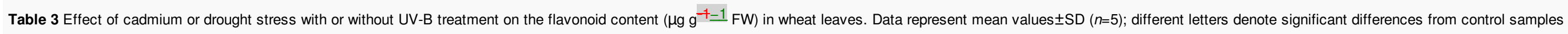
taken on the same day at $p<0.05$.

\begin{tabular}{|c|c|c|c|c|c|c|}
\hline Treatments & Free rutin & Bound rutin & Free myricetin & Bound myricetin & Free quercetin & Bound quercetin \\
\hline CL C & $0.49 \pm 0.01 a$ & $15.35 \pm 8.62 a$ & $0.2 \pm 0.04 a$ & $1.4 \pm 0.16 \mathrm{a}$ & $7.78 \pm 1.13 a$ & $46.84 \pm 11.22 a$ \\
\hline $\mathrm{CL} C d$ & $0.53 \pm 0.22 \mathrm{ab}$ & $22.79 \pm 3.24 a$ & $0.21 \pm 0.1 \mathrm{a}$ & $1.73 \pm 1.26 \mathrm{ab}$ & $8.11 \pm 2.55 \mathrm{a}$ & $55.11 \pm 18.85 a$ \\
\hline CL PEG & $0.73 \pm 0.09 \mathrm{~b}$ & $28.68 \pm 3.94 a$ & $0.49 \pm 0.36 a b$ & $8.57 \pm 0.26 \mathrm{~d}$ & $9.83 \pm 3.2 \mathrm{a}$ & $136.9 \pm 2.17 \mathrm{bc}$ \\
\hline UV C & $1.8 \pm 0.24 \mathrm{c}$ & $41.73 \pm 10.52 b$ & $0.37 \pm 0.15 a b$ & $3.8 \pm 1.13 b$ & $21.96 \pm 5.15 b$ & $140.53 \pm 18.71 \mathrm{c}$ \\
\hline $\mathrm{UV}+\mathrm{Cd}$ & $1.55 \pm 0.12 c$ & $47.35 \pm 11.52 b$ & $0.43 \pm 0.39 a b$ & $7.43 \pm 1.56 \mathrm{~cd}$ & $16.64 \pm 3.91 b$ & $197.36 \pm 33.98 d$ \\
\hline$U V+P E G$ & $1.47 \pm 0.35 \mathrm{c}$ & $40.02 \pm 5.68 b$ & $0.53 \pm 0.08 b$ & $6.15 \pm 0.32 c$ & $21.67 \pm 7.82 b$ & $125.55 \pm 8.12 b$ \\
\hline
\end{tabular}

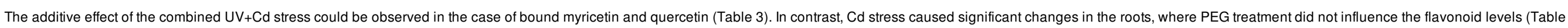

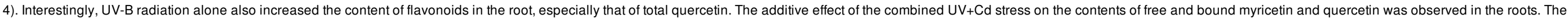
elevated levels of flavonoids were also detected after UV+PEG treatment compared to the individual PEG and UV-B treatments (Table 4). 


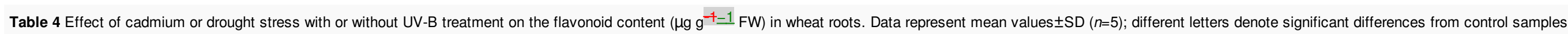
taken on the same day at $p<0.05$.

\begin{tabular}{|c|c|c|c|c|c|c|}
\hline Treatments & Free rutin & Bound rutin & Free myricetin & Bound myricetin & Free quercetin & Bound quercetin \\
\hline CL C & nd & $1.58 \pm 0.54 a$ & $0.41 \pm 0.03 a$ & $1.13 \pm 0.6 \mathrm{a}$ & $6.08 \pm 1.4 a$ & $25.81 \pm 4.2 \mathrm{a}$ \\
\hline $\mathrm{CL} C d$ & nd & $6.81 \pm 1.58 \mathrm{~b}$ & $1.47 \pm 0.42 c$ & $1.95 \pm 0.67 a$ & $15.22 \pm 2.11 \mathrm{~b}$ & $48.72 \pm 3.53 b$ \\
\hline CL PEG & nd & $1.92 \pm 0.19 a$ & $0.42 \pm 0.22 a$ & $1.59 \pm 0.91 a$ & $5.88 \pm 1.19 a$ & $18.21 \pm 4.15 \mathrm{a}$ \\
\hline UV C & nd & $3.7 \pm 2.13 a b$ & $0.85 \pm 0.24 b c$ & $1.95 \pm 0.51 a$ & $15.56 \pm 3.23 b$ & $54.44 \pm 10.43 b$ \\
\hline$U V+C d$ & nd & $6.38 \pm 1.24 b$ & $2.32 \pm 0.17 d$ & $4.6 \pm 0.45 b$ & $42.26 \pm 5.11 \mathrm{c}$ & $76.62 \pm 2.55 \mathrm{c}$ \\
\hline$U V+P E G$ & nd & $4.98 \pm 0.38 b$ & $0.75 \pm 0.07 \mathrm{~b}$ & $4.92 \pm 0.48 b$ & $43.29 \pm 1.55 c$ & $97.53 \pm 12.7 d$ \\
\hline
\end{tabular}

nd: not detected.

\section{Discussion}

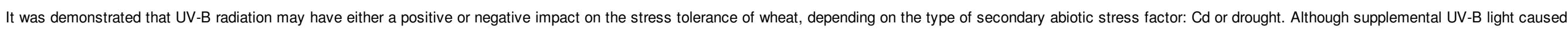

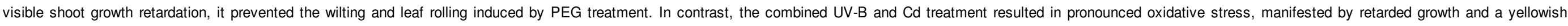
appearance (Kovács et al., 2014).

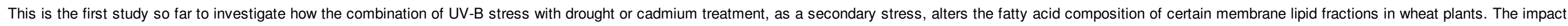
of UV-B radiation on physiological changes has mostly been studied in the leaves, and has not yet been elucidated in the roots, so the data presented here are novel in this respect.

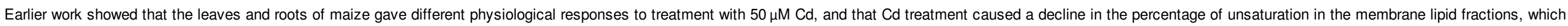

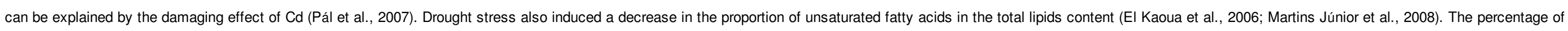

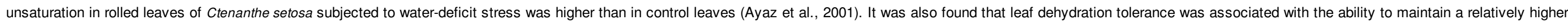

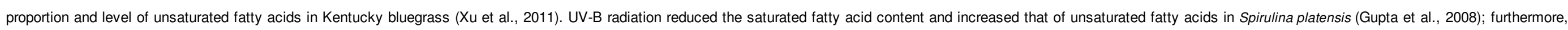

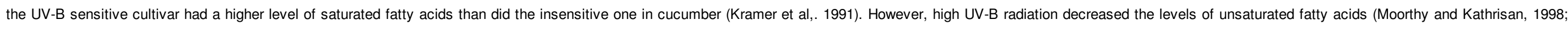
Wulff et al., 1999).

Under the present stress conditions, PEG, Cd and UV-B had different effects on the fatty acid composition in the different membrane fractions; furthermore, in some cases the leaves and roots responded differently.

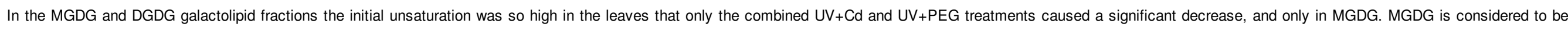

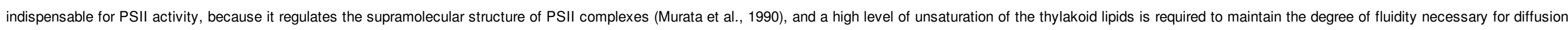

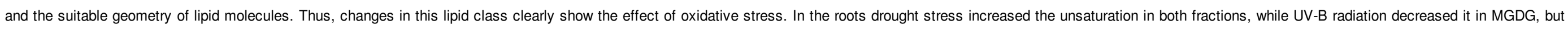

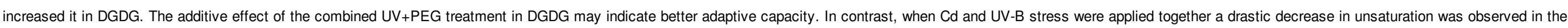
DGDG fraction due to the severe oxidative stress.

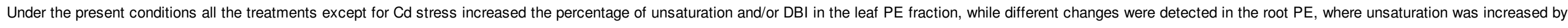

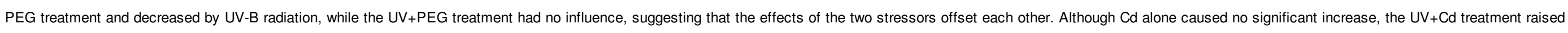
the level of unsaturation.

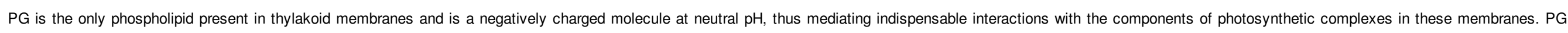

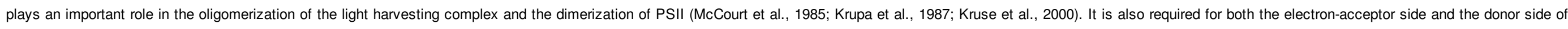

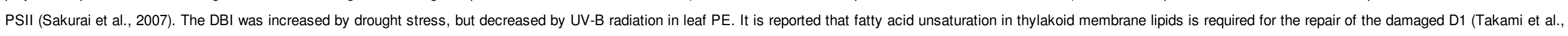




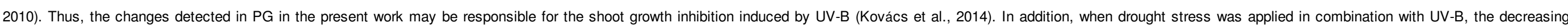

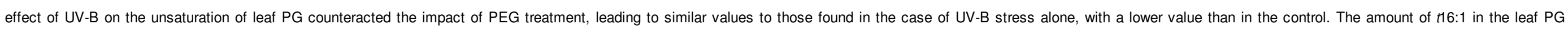

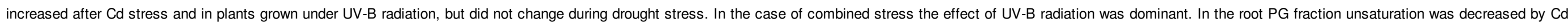
and increased by PEG, while it was not influenced by UV-B. In the case of the combined stresses, the impacts of Cd and PEG prevailed.

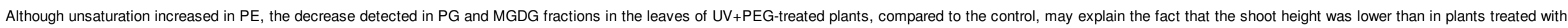

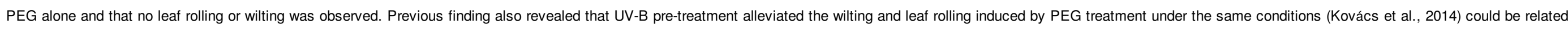

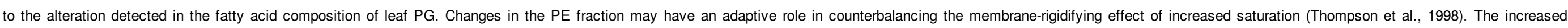

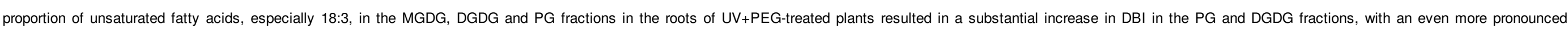

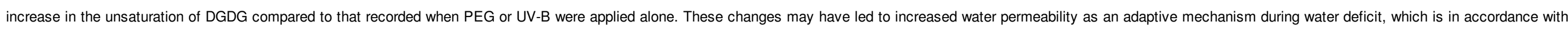
the observation that the relative water content was positively correlated with DBI in Kentucky bluegrass under drought stress (Xu et al., 2011).

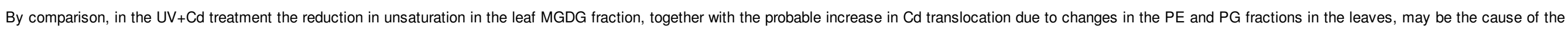

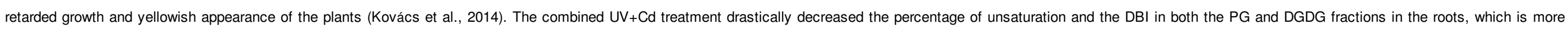

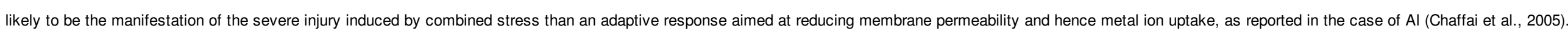

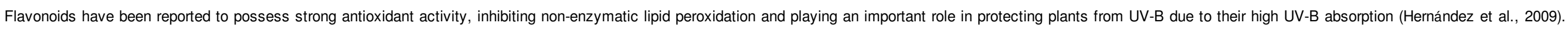

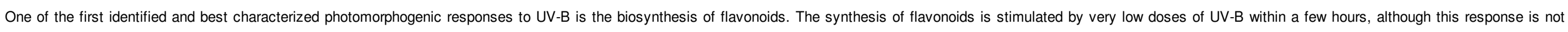

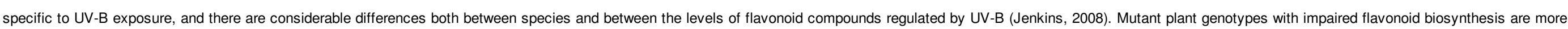

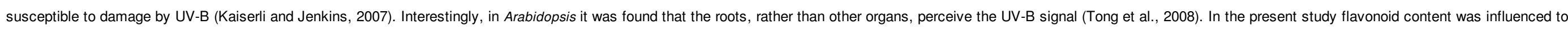

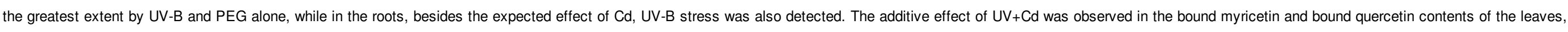

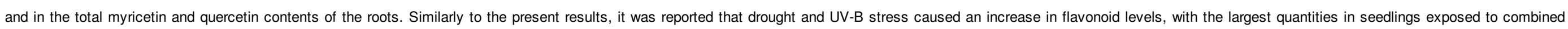

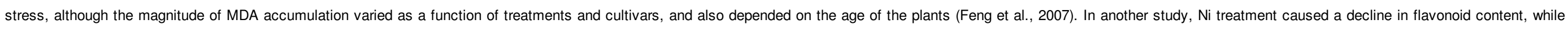

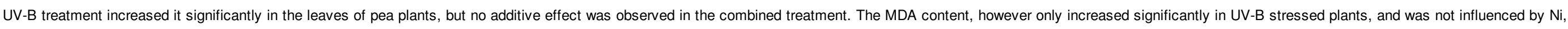

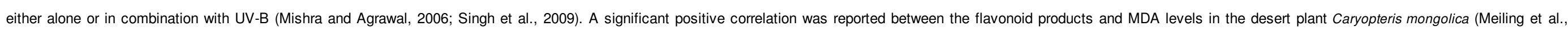

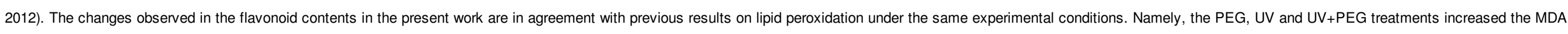

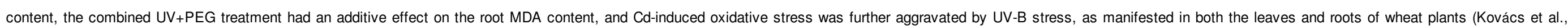
2014).

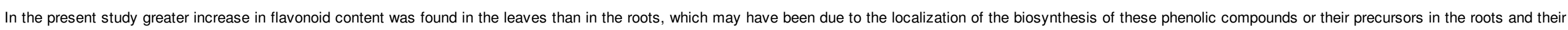

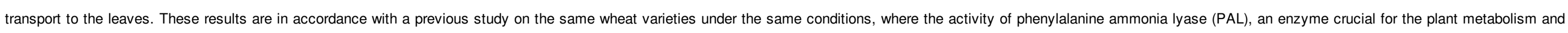

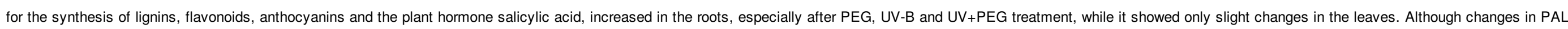

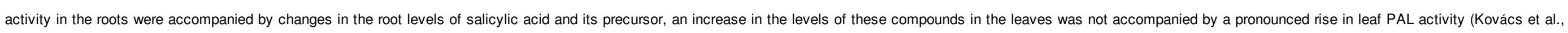
2014).

\section{Conclusion}

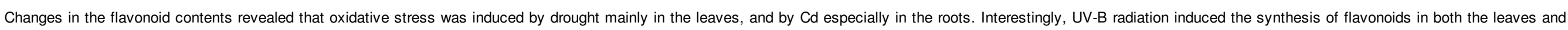

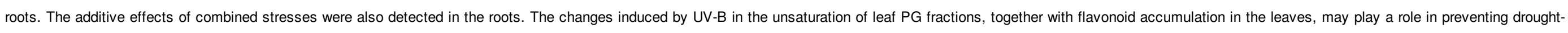

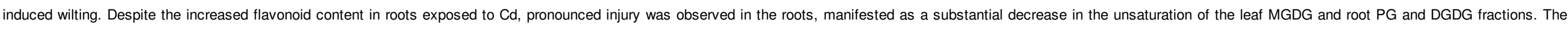
present results suggest a relationship between the capacity of plants to remodel their fatty acid composition and their resistance to various stress factors. 


\section{elsevier_YEESA_5620}

\section{AcknowledgementsAcknowledgments}

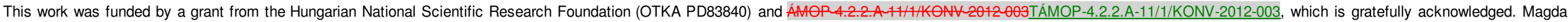
Pál is a recipient of the János Bolyai Scholarship. The authors wish to thank Barbara Hooper for revising the English.

\section{References}

Ayaz A.F., Kadioglu A. and Dogru A., Leaf rolling effects on lipid and fatty acid composition in Ctenanthe setosa (Marantaceae) subjected to water-deficit stress, Acta Physiol. PlantPlant. 23, $2001,43-47$.

Bernat P., Gajewska E., Bernat T. and Wielanek M., Characterisation of the wheat phospholipid fraction in the presence of nickel and/or selenium, Plant Growth Regul. 72, $2014,163-170$.

Bligh E.G. and Dyer W.J., A rapid method of total lipid extraction and purification, Can. J. Biochem. PhysiolPhysiol. 37, 1959, 911-917.

Chaffai R., Marzouk B. and El Ferjani E., Aluminum mediates compositional alterations of polar lipid classes in maize seedlings, Phytememememistry 66, 2005, 1903-1912

El Kaoua M., Serreaj R., Benichou M. and Hsissou D., Comparative sensitivity of two Moroccan wheat varieties to water stress: the relationship between fatty acids and proline accumulation, Bot. Stud. 47, $2006,51-60$.

Feng H., Li S., Xue L., An L. and Wang X., The interactive effects of enhanced UV-B radiation and soil drought on spring wheat, S. Afr. J. Bot. 73, 2007, 429-434.

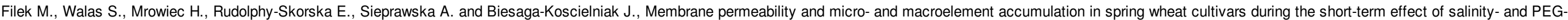
induced water stress, Acta Physiol. PlantPlant. 34, 2012, 985-995.

Gupta R., Bhadauriya P., Chauhan V.S. and Bisen P.S., Impact of UV-B radiation on thylakoid membrane and fatty acid profile of Spirulina platensis, Curr. Microbiol. 56, $2008,156-161$.

Hernández I., Alegre L., Van Breusegem F. and Munné-Bosch S., How relevant are flavonoids as antioxidants in plants?, Trends Plant Sci. 14 (3), 2009, 125-132.

Jenkins G.I., Environmental regulation of flavonoid biosynthesis, In: Givens G.I., Baxter S., Minihane A.M. and Shaw E., (Eds.), Health Benefits of Organic Food: Effects of the Environment, 2008, CABI; Wallingford, 240-262. Kaiserli E. and Jenkins G.I., UV-B promotes rapid nuclear translocation of the Arabidopsis UV-B specific signaling component UVR8 and activates its function in the nucleus, Plant Cell 19, 2007, 2662-2673.

Kovács V., Gondor O.K., Szalai G., Majláth I., Janda T. and Pál M., UV-B radiation modifies the acclimation processes to drought or cadmium in wheat, Env.Environ. Exp. Bot. 100, 2014, 122-131.

Kramer G.F., Norman H.A., Krizek D.T. and Mirecki R.M., Influence of UV-B radiation on polyamines, lipid peroxidation and membrane lipids in cucumber, Phyłtochem.Phytochemistry 30, 1991, 2101-2108.

Krupa Z., Huner N.P.A., Williams J.P., Maissan E. and James D.R., Development at cold hardening temperature. The structure and composition of purified rye light harvesting complex II, Plant Physiol. 84, $1987,19-24$.

Kruse O., Hankamer B., Konczak C., Gerle C., Morris E., Radunz A., Schmid G.H. and Barber J., Phosphatidylglycerol is involved in the dimerization of photosystem II, J. Biol. Chem. 275, 2000, 6509-6514.

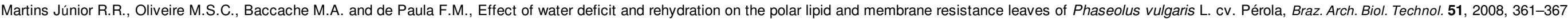

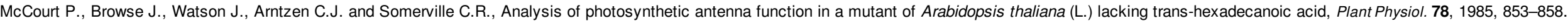
Meuwly P. and Métraux J.P., Ortho-anisic acid as internal standard for the simultaneous quantitation of salicylic acid and its putative biosynthetic precursors in cucumber leaves, Anal. Biochem. 214, 1993, 500-505.

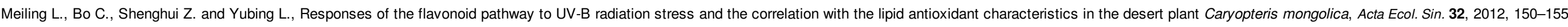

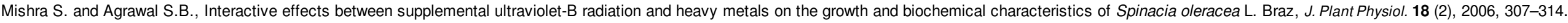

Moorthy P. and Kathrisan K., UV-B induced alterations in composition of thylakoid membrane and amino acids in the leaves of Rhizophora apiculata Blume, Photosynt.Photosynthetica 35, 1998 , 321-328.

Murata N., Higashi S.I. and Fujimura Y., Glycerolipids in various preparations of photosystem Il from spinach chloroplasts, Biochem. Biophys. Acta 1019, 1990, 261-268.

Pál M., Horváth E., Janda T., Páldi E. and Szalai G., Cadmium stimulates the accumulation of salicylic acid and its putative precursors in maize (Zea mays L.) plants, Physiol. PlantPlant. 125, 2005, 356-364.

Pál M., Leskó N., Janda T., Páldi E. and Szalai G., Cadmium-induced changes in the membrane lipid composition of maize plants, Gereat.Cereal Res. Com.Commun. 35, 2007, 1631-1642. 


\section{elsevier_YEESA_5620}

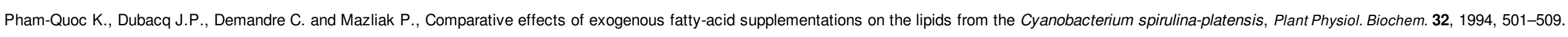

Sakurai I., Mizusawa N., Ohashi S., Kobayashi M. and Wada H., Effects of the lack of phosphatidylglycerol on the donor side of photosystem II, Plant Physiol. 144, 2007, $1336-1346$.

Singh S., Mishra S., Kumari R. and Agrawal S.B., Response of ultraviolet-B and nickel on pigments, metabolites and antioxidants of Pisum sativum L, J. Environ. Biol. 30, $2009,677-684$.

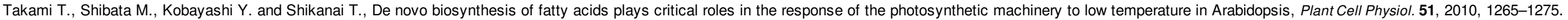

Thompson J.E., Froese C.D., Madey E., Smith M.D. and Hong Y., Lipid metabolism during plant senescence, Prog. tipid.Lipid Res. 37, 1998, 119-141.

Tong H., Leasure C.D., Hou X., Yuen G., Briggs W. and He Z.H., Role of root UV-B sensing in Arabidopsis early seedling development, Proc. Nat.Ae.Natl. Acad. Sci. 105, 2008, 21039-21044.

Wulff A., Anttonen S., Pellinen R., Savonen E.M., Sutinen M.L., Heller W., Sandermann H., Jr and Kangasjärvi J., Birch (Betula pendula Roth.) responses to high UV-B radiation, Boreal Environ. Res. 4, 1999, 77-88.

Xu L., Han L. and Huang B., Membrane fatty acid composition and saturation levels associated with leaf dehydration tolerance and post-drought rehydration in Kentucky bluegrass, Crop Sci. 51, 2011, 273-281.

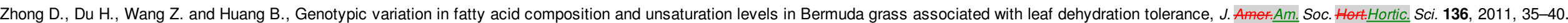
Zlatev Z.S., Lidon F.J.C. and Kaimakanova M., Plant physiological responses to UV-B radiation, Emir. J. Food. AgrieFood Agric. 24, 2012, 481-501.

Highlights

- UV-B influences flavonoid contents and fatty acid composition both in the leaves and roots.

- UV-B has different impact on drought and Cd stress induced changes.

- UV-B radiation might have either a positive or negative impact under the same conditions.

\section{Queries and Answers}

Query:

Please confirm that given names and surnames have been identified correctly and are presented in the desired order.

Answer: It has been checked.

Query:

The country name has been inserted for the affiliation. Please check, and correct if necessary.

Answer: It has been checked. 